\title{
EL PEZ MESOPELAGICO CENTROLOPHUS NIGER (GMELIN, 1789) (PISCES: PERCIFORMES) Y SU HALLAZGO EN AGUAS DEL TALUD CONTINENTAL CHILENO SITUADAS SOBRE LA PLACA SUDAMERICANA
}

\author{
MESOPELAGIC FISH CENTROLOPHUS NIGER (GMELIN, 1789) \\ (PISCES: PERCIFORMES) RECORDED IN CHILEAN CONTINENTAL SLOPE \\ WATERS OF SOUTH AMERICAN PLATE
}

\author{
Pablo Reyes ${ }^{1}$, Mathias Hüne ${ }^{2} \&$ Víctor H. Ruiz $^{3}$ \\ ${ }^{1}$ Falkland Islands Government, Fisheries Department. P.O. Box 598, F.I.P.A.S.S. Stanley, Falkland Island. \\ Email: preyes@fisheries.gov.fk \\ ${ }^{2}$ Universidad Austral de Chile. Escuela de Biología Marina. Casilla 567, Valdivia. Chile. \\ ${ }^{3}$ Universidad de Concepción. Departamento de Zoología. Casilla 160-C, Concepción. Chile.
}

\begin{abstract}
RESUMEN
Se estudió un ejemplar de Centrolophus niger (Gmelin, 1789) (Perciformes: Centrolophidae), capturado como fauna acompañante en faenas de pesca de "besugo" Epigonus crassicaudus de Buen, 1959 el 14 de octubre del año 2003 frente a la provincia de Valdivia $\left(39^{\circ} 04^{\prime} \mathrm{S} ; 7^{\circ} 57^{\prime} \mathrm{W}\right)$ a una profundidad de $400 \mathrm{~m}$, mediante pesca de arrastre de media agua. Este constituye el primer registro para la especie en aguas del talud continental chileno situadas sobre la Placa Sudamericana.
\end{abstract}

Palabras claves: Arrastre de media agua, fauna acompañante, Centrolophidae, ictiofauna chilena, Chile.

\section{ABSTRACT}

A specimen of Centrolophus niger (Gmelin, 1789) (Perciformes: Centrolophidae), caught as by catch of the "chilean cardenalfish" Epigonus crassicaudus de Buen 1959, was studied. The specimen was caught on October 14,2003 , on the continental slope just off shore Valdivia Province $\left(39^{\circ} 04^{\prime} \mathrm{S} ; 73^{\circ} 57^{\prime} \mathrm{W}\right)$, at $400 \mathrm{~m}$ deep, through midwater trawler fishing. This one is the southernmost record of this genus and species, being simultaneously the first record in Chilean continental slope waters of South American Plate, and the second record for the Chilean ichthyofauna.

Keywords: Midwater trawler fishing, by catch, Centrolophidae, Chilean ichthyofauna, Chile.

\section{INTRODUCCION}

El estudio de la composición de especies en un ecosistema determinado es de gran importancia para la realización de posteriores trabajos en las distintas áreas de la ciencia como biología evolutiva, ecología, biogeografía y conservación (Eastman 2005). En lo anterior radica la inquietud de aumentar el conocimiento y complementar la información sobre la taxonomía de peces, base fundamental de la zoogeografía, disciplina encargada de abordar el origen y la distribución actual de las especies, mediante la búsqueda de los centros de origen de los diferentes taxa con el objetivo de estudiar su dispersión hasta alcanzar su distribución actual (Tellenía 1991). Este campo ha despertado el interés de numerosos especialistas con el propósito de responder interrogantes de la 
ictiofauna marina en determinados lugares, sus adaptaciones ecológicas a los diferentes ambientes en los que viven y los mecanismos de especiación que les han permitido colonizar ambientes cada vez más restrictivos. De hecho, actualmente, existe un gran interés por aumentar nuestro conocimiento de la abundancia, distribución y sobre todo, la diversidad de la vida marina en los océanos del mundo; con el fin, entre otros, de dilucidar los cambios en la biodiversidad global a causa de la pesca, la contaminación y el cambio climático (O’ Dor \& Yarincik 2003).

Así, en los últimos veinte años ha existido un gran cambio en relación con la composición taxonómica de la ictiofauna de Chile, especialmente la marina, donde ha existido una serie de contribuciones que han contribuido a modificar los conceptos sistemáticos, zoogeográficos, ecológicos y pesqueros sobre los peces. El valor de los diferentes reencuentros (Moreno \& Jara 1981; Ojeda 1983; Pequeño 1978, Pequeño, 1981; Pequeño et al. 1991; Pequeño 2004) y nuevos registros (Lamilla 1986; Lamilla \& Pequeño 1999; Morrison \& Pequeño 2003; Pequeño 1978a; 1991; Pequeño et al. 1992) ha permitido estructurar el conocimiento científico de la ictiofauna chilena, compuesta en la actualidad por cerca de 1.100 especies (Pequeño 1989 y 1997), a las cuales se siguen sumando nuevas especies a medida que se desarrollan cruceros oceanográficos en aguas de la Zona Económica Exclusiva (ZEE) y a medida que se inician prospecciones de nuevos recursos, que conllevan la exploración de nuevos caladeros (Nakamura etal.1986; Meléndez 1999).

En la presente investigación se adhiere al llamado realizado por Pequeño (1989) sobre la necesidad de informar los nuevos registros como forma de contribuir a mejorar el conocimiento de los peces de Chile y del Pacífico sur oriental, presentando una adición a la ictiofauna chilena; Centrolophus niger (Gmelin 1789) (Perciformes: Centrolophidae).

\section{MATERIALES Y METODOS}

Un ejemplar de pez óseo perteneciente al Orden Perciformes colectado el día 14 de octubre del año 2003 en la cámara de frío de la Pesquera Grimar, planta Coronel, Región del Bío-Bío, proveniente de faenas de pesca comercial de "besugo" E. crassicaudus efectuadas a $400 \mathrm{~m}$ de profundidad en aguas de la
Región de los Ríos (3904` S; 73 57` W) por el buque arrastrero "Don Enrique". Tras el hallazgo, el ejemplar fue fijado en formalina $10 \%$ y trasladado al Instituto de Zoología de la Universidad Austral de Chile (IZUA), donde se procedió a realizar su identificación siguiendo a Haedrich (1967). El ejemplar fue entregado al Museo Nacional de Historia Natural de Chile y catalogado con el número de colección: MNHNC P 7250.

Las abreviaturas usadas en el texto corresponden a: AMC: altura máxima de cuerpo; D: distancia; DPA: distancia pre anal; DPO: distancia post ocular; DPR: distancia pre ocular; DPT: distancia post anal; L: longitud; LBA: longitud base aleta anal; LBD: longitud base aleta dorsal; LBP: longitud base aleta pectoral; LC: longitud de la cabeza; L.E.: Longitud estándar; L.T.: Longitud total; S.L.: Standard length, IZUA: Instituto de Zoología, Universidad Austral de Chile; MNHNC P: Museo Nacional de Historia Natural de Chile, Peces.

\section{RESULTADOS}

Material Examinado. MNHNC P7250, $92 \mathrm{~cm}$ L.E. Centrolophus niger (Gmelin 1789) (Fig. 1).

CARACTERES Diagnósticos. La especie $C$. niger presenta cuerpo elongado, cuya máxima altura rara vez excede el $30 \%$ de la longitud estándar. Musculatura firme. Pedúnculo caudal largo. Aleta dorsal se origina levemente detrás del nivel de la inserción de las aletas pectorales. Ano y poro genital en la parte media ventral del cuerpo. Aleta anal se origina levemente detrás de la parte media del cuerpo. Aletas pectorales redondeadas en juveniles, puntiaguda en adultos. Aletas pélvicas insertas bajo la porción posterior de la base de las aletas pectorales y conectadas con el abdomen mediante pequeñas membranas. Cuerpo cubierto con escamas cicloideas, pequeñas. Línea lateral arqueada anteriormente y extendida por el pedúnculo caudal. La cabeza usualmente menos que el $25 \%$ de la longitud estándar. Ojo de moderado tamaño, sin tejido adiposo. Nariz redondeada. Angulo de laboca proyectado bajo el margen del ojo. Premaxilar no protráctil. Mandíbulas con dientes pequeños, puntiagudos, uniseriados y espaciados, su número aumenta según la longitud del pez. Opérculo y preopérculo con márgenes finamente denticulados. Siete branquiostegales, cinco ceratohiales, dos epihiales. 


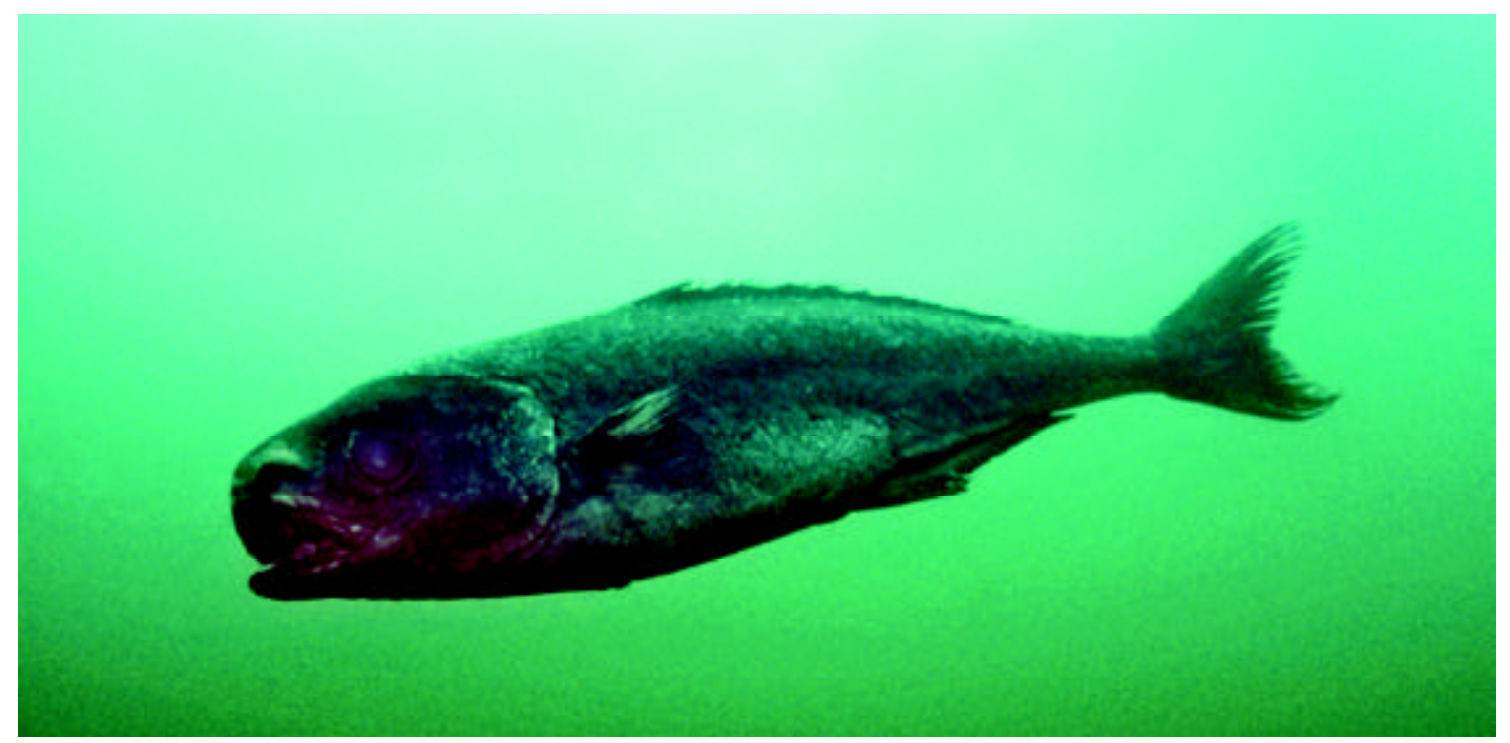

FIGURA 1. Centrolophus niger MNHNC P72484 (78 cm L.E). Imagen de Mathias Hüne @ $).$

FIGURE 1. Centrolophus niger MNHNC P72484 (78 cm S.L). Mathias Hüne (C) Image.

El principal carácter diagnóstico que diferencia a Centrolophus de otros géneros de Stromateoideos es que presenta de 160 a 230 escamas en la línea lateral.

DESCRIPCIÓN. El espécimen, un ejemplar de $920 \mathrm{~mm}$ de L.T., presenta cuerpo elongado, musculatura firme; pedúnculo caudal largo; su aleta dorsal se origina levemente detrás del nivel de la inserción de las aletas pectorales; el ano y el poro genital se encuentran en la parte media ventral del cuerpo; la aleta anal se origina levemente detrás de la parte media del cuerpo; las aletas pectorales son puntiagudas; aletas pélvicas se encuentran insertas bajo la porción posterior de la base de las aletas pectorales y conectadas con el abdomen mediante pequeñas membranas; el cuerpo se encuentra cubierto con escamas cicloideas, pequeñas; línea lateral arqueada anteriormente y extendida por el pedúnculo caudal; ojo de moderado tamaño y sin tejido adiposo; nariz redondeada; ángulo de la boca se proyecta bajo margen del ojo; premaxilar no protráctil; mandíbulas con dientes pequeños, puntiagudos, uniseriados y espaciados; opérculo y preopérculo con márgenes finamente denticulados.

Morfometría. Altura máxima del cuerpo corres- ponde a un $33.9 \%$ de la L.E.; la longitud de la cabeza corresponde a un $26.3 \%$ de la L.E.; la distancia pre anal corresponde a un $53.8 \%$ de la L.E.; la distancia post anal corresponde a un 76.9 $\%$ de la L.E.; la longitud de la base de la aleta dorsal corresponde a un $52.1 \%$ de la L.E.; la longitud de la aleta pectoral corresponde a un $13.5 \%$ de la L.E.; la longitud de la aleta anal corresponde a un $28.9 \%$ de la L.E.; el diámetro ocular corresponde a un $5.1 \%$ de la L.E.; la distancia post ocular corrresponde a un $100.1 \%$ de la L.E.; la pre ocular corresponde a un $7.7 \%$ de la L.E.; Las características morfométricas se presentan en la Tabla I.

Color en formalina $10 \%$. Cuerpo y aletas homogéneamente plomas.

DistRIBUCIÓN GEOGRÁFICA . Especie mesopelágica, de amplia distribución geográfica. En el hemisferio sur es conocida en Australia, Nueva Zelanda, Sudáfrica (Haedrich, 1986) y Argentina (Wöhler et al. 1999). En Chile se le ha visto en las cercanías de Juan Fernández (IFOP y UACh 2003). También se le encuentra en el Atlántico norte y sur, en el Indico y en el mar Mediterráneo, a profundidades de 250 a 1.100 metros (Haedrich 1990). 
TABla I. Datos morfométricos de Centrolophus niger MNHNCP7250.

TABLE I. Morphometric data of Centrolophus niger MNHNCP7250.

\begin{tabular}{lcc}
\hline Medidas & Longitud $(\mathrm{cm})$ & \% L.E. \\
\hline L.T. & 92.0 & 117.9 \\
L.E. & 78.0 & 100 \\
AMC & 26.5 & 33.9 \\
LC & 20.5 & 26.2 \\
DPA & 42.0 & 53.8 \\
DPT & 60.0 & 76.9 \\
LBD & 40.6 & 52.1 \\
LBP & 10.5 & 13.4 \\
LBA & 22.5 & 28.8 \\
DO & 4.0 & 5.1 \\
DPO & 82.0 & 105.1 \\
DPR & 6.0 & 7.6 \\
\hline
\end{tabular}

\section{DISCUSION}

La familia Centrolophidae (Osteichthyes: Perciformes) se encuentra representada en aguas chilenas por cuatro géneros y nueve especies. Dichas especies son: Seriolella caerulea Guichenot, 1848; Seriolella porosa Guichenot 1848; Seriolella punctata (Bloch y Schneider, 1801); Seriolella violacea Guichenot, 1848; Icichthys australis Haedrich, 1966; Schedophilus huttoni (Waite 1910); Schedophilus labyrinthicus McAllister y Randall, 1975 (Pequeño, 1989); Schedophilus griseolineatus (Norman 1937) (Venegas y Sielfeld 1998); y Centrolophus niger (Gmelin 1789) (IFOP y UACh, 2003). Las primeras cuatro especies constituyen recursos pesqueros para Chile (Boré et al. 1984).

El primer registro de $C$. niger en Chile se produce el año 2003 como resultado del proyecto "Investigación Situación Pesquería Demersal Centro-Sur', desarrollado por el Instituto de Fomento Pesquero (IFOP) y la Universidad Austral de Chile (UACh), quienes notifican su presencia como fauna acompañante en la pesquería de "orange roughy", Hoplostethus atlanticus Collet, 1889 y "alfonsino" Beryx splendens Lowe, 1834 (IFOP y UACh, 2003), en montes submarinos de la Placa de Naz- ca, sin embargo, en tal publicación no se detalla la ubicación geográfica de la captura, razón por la cual se considera que el presente es el primer registro documentado en Chile.

La presencia de $C$. niger ha sido notificada en un vasto sector oceánico, sin embargo, considerando sólo sus registros en el Océano Pacífico sur, que incluyen desde el sector occidental (Lawson 2001) hasta el sector oriental (IFOP y UACh, 2003) se evidencia la capacidad que presenta la especie para realizar movimientos longitudinales y latitudinales amplios. Una explicación a esta movilidad es su condición de pez mesopelágico (Haedrich 1967), la cual le permitiría realizar grandes desplazamientos oceánicos, aprovechando las condiciones térmicas estables que imperan en aguas profundas (Moreno y Castila 1977). Por ello, la presencia de este pez mesopelágico en aguas que se encuentran en las cercanías de la costa continental chilena puede vincularse a la geomorfología continental, caracterizada por presentar una plataforma continental angosta, que permite a los peces mesopelágicos aproximarse a pocas millas de la costa, sin abandonar el talud continental (Pequeño 2000).

La presencia de la especie en el paralelo $39^{\circ} \mathrm{S}$ no implica necesariamente que el ejemplar haya perdido el rumbo al llegar al talud continental de la Región de los Ríos, Región de Chile, pues su distribución geográfica es antitropical (Haedrich 1990) y vinculada a los extremos australes de los continentes del Hemisferio Sur (Haedrich 1967). La falta de registros previos de $C$. niger en el sector podría deberse más bien a la falta de conocimiento ictiológico por parte de los biólogos que trabajan embarcados y al personal de las plantas de procesamiento, incapaz finalmente de informar los registros. Por ello, el hallazgo de un ejemplar de $C$. niger en el talud continental de la zona centro sur de Chile no debería ser tratado como un hecho esporádico. Según informa el jefe de producción de la planta pesquera Grimar de Coronel, quien cedió el ejemplar al que denominaba vernacularmente "cojinova negra", anteriormente se han observado ejemplares similares, vinculados a la fauna acompañante de "besugo" $E$. crassicaudus y "merluza de cola" Macruronus novaezelandiae magellanicus Lönnberg 1907, ambas pesquerías efectuadas con redes de arrastre sobre el talud continental de la zona centro sur de Chile (Jorge Rosas. Com. Pers.). 
En conclusión, el reencuentro de $C$. niger confirma la presencia del género y la especie en aguas marinas de la Zona Económica Exclusiva (ZEE) de Chile y documenta por primera vez la presencia de la especie en aguas marinas chilenas colindantes al continente, situadas sobre la Placa Sudamericana.

\section{AGRADECIMIENTOS}

Al Sr. Jorge Rosas, jefe de planta de Pesquera Grimar Coronel, por facilitar el material de estudio. A M. Angélica de la Maza, Soledad y Francisca Alvarez y Gerardo Hüne por sustentar nuestra logística en Coronel y Concepción. Al Dr. Germán Pequeño del Instituto de Zoología de la Universidad Austral de Chile por colaborar en la identificación del ejemplar estudiado. A los investigadores de la Universidad Austral de Chile, Dr. Germán Pequeño, Dr. Carlos Moreno y MSc. Alejandro Bravo, por sus correcciones, comentarios y aportes bibliográficos. A tres evaluadores anónimos por sus valiosos comentarios.

Esta publicación es resultado parcial del proyecto FDI-MINEDUC "Guía de Peces del Sur de Chile" del Ministerio de Educación de Chile, auspiciada por la Dirección de Investigación y Desarrollo (DID) de la Universidad Austral de Chile (UACh), Escondida, Marine Harvest, Kodak, Navimag, Socovesa, Antártica y patrocinada por la Dirección de Asuntos Estudiantiles y Escuela de Biología Marina, ambas de la Universidad Austral de Chile, Corporación pro Defensa de la Flora y Fauna de Chile (CODEFF) y Fondo Mundial para la Naturaleza (WWF).

\section{BIBLIOGRAFIA}

Boré, D., N. Henríquez \& G. Espinoza. 1984. Chile: Sus recursos pesqueros. Corporación de Fomento de la Producción. Instituto de Fomento Pesquero. Santiago, Chile. 160 pp.

Eastman, J. 2005. The nature of the diversity of Antarctic fishes. Polar Biology 28(2): 93-107.

HaEdRICH, R. 1967. The Stromateoid Fishes: Systematics and a Classification. Bulletin of the Museum of Comparative Zoology 135 (2): 62-69.

HAEDRICH, R. 1986. Stromateidae. p. 842-846. In M.M. Smith and P.C. Heemstra (eds.) Smiths' sea fishes. Springer-Verlag, Berlin.

Haedrich, R. 1990. Centrolophidae. In J.C. Quero, J.C. Hureau, C. Karrer, A. Post and L. Saldanha (eds.) Check-list of the fishes of the eastern tropical
Atlantic (CLOFETA). JNICT, Lisbon; SEI, Paris; and UNESCO, Paris, 2: 1011-1013.

Instituto de Fomento Pesquero (IFOP) y Universidad Austral de Chile (UACH). 2003. Catálogo fauna íctica asociada a la pesquería chilena de orange roughy (Hoplostethus atlanticus). Subsecretaría de Pesca. Valparaíso. 52 pp.

Lamilla, J. 1986. Bathyraja albomaculata (Norman 1937): Primer registro para las costas de Chile (Chondrichthyes, Rajidae). Boletín de la Sociedad de Biología de Concepción 57: 177-182.

Lamilla, J. \& G. PequeÑo. 1999. Descripción esquelética comparativa y primer registro en el Océano Pacífico de Bathyraja multispinis (Norman, 1937) (Rajiformes; Rajoidei), con referencia a otras especies de Bathyraja. Revista de Biología Marina y Oceanografía 34(2): 281-290.

Lawson, T. 2001. Observer data held by the oceanic fisheries programmer covering tuna fishery by catches in the western and central Pacific Ocean. SCTB14 Working Paper, SWG-9. Oceanic Fisheries Programmer. Secretariat of the Pacific Community. Noumea, New Caledonia. 42 pp.

Meléndez, R. 1999. Nuevas adiciones a la ictiofauna mesopelágica y demersal entre isla Mocha y la península de Taitao $\left(38^{\circ} 04^{\prime}-46^{\circ} 13^{\prime} \mathrm{S}\right)$, Chile. Revista de Biología Marina y Oceanografía 34(2): 139-143.

Moreno, C. \& F. JARA. 1981. Reencuentro de Notothenia trigramma Regan en los canales sur patagónicos, Chile (Pisces: Nototheniidae). Noticiero Mensual del Museo Nacional de Historia Natural de Chile 26 (301-302): 7-9.

Moreno, C. \& J. Castilla. 1977. Guía para el reconocimiento y observación de Peces de Chile. Serie Expedición a Chile. Ed. Gabriela Mistral. Santiago. 119 p.

Morrison, E. \& G. Pequeño. 2003. Primer registro del Zoárcido Crossostomus sobrali (Lloris y Rucabado 1987) (Osteichthyes, Zoarcidae) en el Estrecho de Magallanes, Distrito fueguino, Chile. Anales del Instituto de la Patagonia, Punta Arenas, 31: 105-109.

NaKamura, I., T. Inada, M. Takeda \& H. Hatanaka. 1986. Important fishes trawled off Patagonia. Japan Marine Fishery Resource Research Center, Tokyo. $369 \mathrm{p}$.

O'Dor, R. \& K. Yarincik. 2003 The Census of Marine Life: Understanding marine biodiversity-past, present and future. Gayana 67 (2): 145-152.

OJEDA, P. 1983. Distribución latitudinal y batimétrica de la ictiofauna demersal del extremo austral de Chile. Revista Chilena de Historia Natural 56: 61-70.

Pequeño, G. 1978a. Doce nuevos registros de peces para la costa de Valdivia, Chile, y su alcance ictiogeográfico. Revista de la Comisión Permanente del Pacífico Sur 9:109-126.

Pequeño, G. 1978b. Presencia de Notothenia wiltoni Regan, 1913 al norte de los canales de Chiloé, Chile (Teleostomi, Nototheniidae). Noticiero 
Mensual del Museo Nacional de Historia Natural de Chile 22(262): 3-6.

Pequeño, G. 1981. The rare fish Neophrynichthys marmoratus Reagan 1913, in the southern slope of Chile (Teleostomi, Psychrolutidae). Journal of Natural History 15:965-970.

Pequeño, G. 1989. Peces de Chile, Lista Revisada y Comentada. Revista de Biología Marina, Valparaíso, 24 (2): 1-132.

Pequeño, G. 1991. Nuevos registros de Trachipterus trachipterus (Gmelin, 1789) frente a Valdivia (Osteichthyes, Trachipteridae). Boletín de la Sociedad de Biología de Concepción 62: 119-122.

Pequeño, G. 1997. Peces de Chile, Lista Revisada y Comentada: Addendum. Revista de Biología Marina y Oceanografía 32(2): 77-94.

Pequeño, G. 2000. Delimitaciones y relaciones biogeográficas de los peces del Pacifico Suroriental. Estudios Oceanológicos 19: 53-76.

Pequeño, G. 2004. Nemadactylus bergi (Norman 1937) frente a Bahía Mansa, Chile (Osteichthyes:
Cheilodactylidae). Ciencia y Tecnología del Mar 27(1): 121-125.

Pequeño, G., A. Cea \& W. Sielfeld. 1992. Primer registro en Chile para tres especies de peces teleósteos marinos, en base a fotografías. Boletín de la Sociedad de Biología de Concepción 63: 169-173.

Pequeño, G., J. Lamilla \& A. Crovetto. 1991. Captura de Somniosus cf. pacificus Bigelow y Schroeder, 1944, frente a Valdivia, Chile, con notas sobre su contenido gástrico (Chondrichthyes, Squalidae). Estudios Oceanológicos 10:117-122.

Tellería, J. 1991. Zoología evolutiva de los vertebrados. Ed. Sintesis. Madrid 168 p.

Venegas, C. \& W. Sielfeld. 1998. Catálogo de los vertebrados de la Región de Magallanes y Antártica chilena. Ed. Universidad de Magallanes. Punta Arenas. 122 p.

Wöhler, O.A., S. Giussi, F. García, J. Sánchez, H. Hansen, H. Cordo, G. Alvarez, S. Incorvaia, R. Reta \& V. Abachian. 1999. Resultados de la campaña de evaluación de peces demersales australes efectuada en el verano de 1997. INIDEP Informe Técnico 24. Mar del Plata, Argentina. 70 pp. 